maßen vergleichen könnte. Es ist der ganze Riss davon unentlehnt, unversucht und neu, die Ausarbeitung aus einer scharfen Untersuchung von acht tausend Pflanzen genommen... Aber was Linnaeus getan, hat niemand versucht, noch getan.» Et le Hollandais H. Borhaave, professeur de botanique à Leyde, écrit dans une lettre adressée à Linné le 13 janvier 1737, à propos du même ouvrage: «Secula laudabunt, boni imitabuntur, omnibus proderit.»

A la fin de la brochure, on trouve quelques critiques anonymes, tirées de revues scientifiques, ainsi qu'une liste bibliographique renvoyant le lecteur à d'autres témoignages sur les ouvrages de Linné.

Mais revenons à la liste biographique contenue au début de la brochure. Elle commence comme suit: «1707 Maji 13 natus». C'est là, comme le fait déjà remarquer Flatt, ${ }^{12}$ l'indication la plus authentique de la date de naissance de Linné! Linné suivait le calendrier julien. A cette époque (jusqu'en 1800), la différence entre les calendriers julien et grégorien était de 11 jours. Linné, donc, d'après le nouveau calendrier, est né le 24 mai 1707 - et non pas le 23 mai comme l'indiquent la plupart des auteurs.

Il ne nous semblait pas inutile d'attirer l'attention des naturalistes sur ce fait, déjà constaté par Flatt (l. c.), dont l'article paraît être resté peu connu.

Ajoutons que l'attaque de Wallerius, qui a provoqué en première ligne cette publication rarissime, n'eut pas de succès: le 5 mai 1741 , Linné fut nommé professeur à l'académie d'Upsal et succéda ainsi à Roberg.

A. Becherer.

\title{
Eine Vorlesung über die Symphysentrennung von Rudolf Abraham Schiferli (1775-I837)*
}

Auf die Idee, durch Trennung der beiden Schambeine in der Symphyse unter der Geburt eine gewisse Erweiterung des weiblichen Beckens zu

12 Flatt 1. c. 1902 , p. 83.

* Biographische Notiz: Rudolf Abraham Schiferli wurde am 30. September 1775 in Thun geboren und starb am 3. Juni 1837 in Bern. Er studierte in Bern und Jena, wo er 1795 zum Doktor der Medizin und Chirurgie promovierte. Zur weiteren Ausbildung ging er nach Paris, wo er unter Boyer und Larrey, dann nach Wien, wo er unter Johann Peter Frank arbeitete. In seine Heimat zuruckgekehrt, erwarb er sich bald den Ruf eines aus- 
erreichen, ist man wohl dadurch gekommen, daß hie und da bei spontanen Geburten, aber auch bei Extraktionen die Symphyse nachgibt und zerreißt. Im letzteren Falle mag man dabei eine fühlbare Erleichterung der Extraktion bemerkt haben; so lag denn der Gedanke nahe, das, was die Natur tat, nachzuahmen und die Symphyse durch einen Schnitt zu trennen in der Absicht, den geraden Durchmesser des Beckeneinganges dadurch zu erweitern.

Schiferli trägt in seiner Vorlesung die Technik der Symphyseotomie oder wie er sie nennt, der Synchondrotomia ossium pubis vor, weil, wie er sagt, man diese Operation kennen müsse; er selber aber lehnt ihre Ausführung ab.

Die Idee der Schambeintrennung zur Erweiterung des knöchernen Beckens führt Schiferli zurück auf die von einigen gemachte, von anderen geleugnete Erfahrung, daß während der Schwangerschaft die zwischen Schamknochen, den Darmbeinen und dem Heiligenbein (os sacrum) befindliche Substanz zunehme, mithin die Beckenhöhle erweitert werde. Beispiele beweisen ihm, daß diese Operation mit glücklichem Erfolge vorgenommen werden könne; doch zeigen eine Menge anderer Beobachtungen, daß sie noch öfter einen höchst traurigen Ausgang nehme; der geringe Nutzen der Schambeintrennung stehe erfahrungsgemäß in keinem vorteilhaften Verhältnis zu ihren Nachteilen.

Als Indikation zu ihrer Ausführung verzeichnet Schiferli:

1. Die Einklemmung des Kopfes so, daß er sich nicht auf- noch abwärts bewegen lasse, bei lebendem Kinde und der Unmöglichkeit der Zangenapplikation. Meist könne aber der Kopf nach gemachtem Kaiserschnitt doch aufwärts bewegt werden.

2. Wenn bei lebendem Kinde bei engem Becken weder Zange noch Wendung tunlich sei und der Gebärmutter schon Entzündung, Brand oder Zerreißung drohe. Auch hier würde er lieber noch den Kaiserschnitt wagen, da für die Mutter die Gefahr nicht kleiner sei.

3. Wenn man überzeugt sei, daß bei einer Erweiterung des geraden

gezeichneten Arztes. Im Frühjahr 1799 ernannte ihn das helvetische Direktorium zum Oberfeldchirurgen, als solcher machte er den Krieg von 1799 mit. Später erhielt er den Titel eines Generalinspektors der Gesundheitspflege der helvetischen Truppen. (Vergl. den von Alfred Rufer, Archivar in Bern, herausgegebenen sehr interessanten Bericht Schiferlis an den helvetischen Senat, aus dem Jahre 1802, über die Organisation des Medizinalwesens der helvetischen Truppen; Schweiz. med. Wschr. 1929, 1311) - Schiferli war auch einer der Gründer und Lehrer am medizinischen Institut in Bern und Mitbegründer der medizinisch-chirurgischen Gesellschaft des Kantons Bern. 
Durchmessers von drei Linien $(6,34 \mathrm{~mm})$ der Durchgang des Kopfes möglich scheine.

Aber auch hier hält Schiferli den Nutzen der Operation für problematisch, weil selbst der geschickteste Geburtshelfer nicht mit Bestimmtheit wissen könne, ob nicht der Kopf des Kindes absolut zu groß sei und mithin nach der Trennung doch nicht durchgebracht werde.

Als weitere Nachteile zählt er auf:

1. Die Möglichkeit, daß durch den Eingriff eine Erweiterung des Beckens doch nicht eintrete, z. B. bei zu kurzen übrigen Durchmessern, bei schiefem (also schrägverengtem) Becken, z. B. dann, wenn durch die Drehung der Darmbeine das Kreuzbein stärker nach vorne tritt (also bei rhachitisch plattem Becken, wie wir es heute nennen).

2. Häufiges Auftreten von Entzündung, Eiterung, Brand, Lähmung und Beinfraß als Folge der gewaltsamen Trennung der Ligamente und des Zellgewebes der Blase.

Die Operation werde darum selten gemacht und Schiferli rät davon $a b$, weil er sie für entbehrlich hält; doch müsse der Chirurg sie kennen, weshalb er sie auch beschreibe.

Als Vorbereitung sind etwa die selben Gerätschaften bereit zu halten, wie für den Kaiserschnitt. Dazu kommt noch ein dünnes, gewölbtes Scalpell, eine Säge und ein breiter lederner mit Kissen belegter Gürtel.

In einem Zuge werden dann in der Mittellinie vom oberen Rande des Schambeines aus die Weichteile bis nahe an den unteren Rand desselben durchtrennt, dann die Symphyse genau in der Mitte langsam durchschnitten, während Gehülfen die Darmbeine gegen einander halten, um ein plötzliches Auseinanderspringen derselben zu verhüten, was große Verletzungen und Zerreißungen zur Folge haben würde. Ist die Symphyse verknöchert, was hie und da vorkommt, so muß die Säge die Trennung ausführen.

Gewöhnlich treten die Knochen zwei und einen halben Zoll auseinander (zirka 6,75 cm); wenn nicht, so kann man dies verursachen durch auseinanderhalten der gebogenen Schenkel. Die zwei und ein halb Zoll können nur mit Mühe den geraden Durchmesser um drei Linien verlängern $(6,34 \mathrm{~mm})$.

Dann wird die Geburt beendigt, durch den angelegten Gürtel, die Knochenwunde zusammengebracht und die Wunde unter der strengsten Ruhe als einfache Schnittwunde behandelt.

Wie wir sehen, ist die von Schiferli vorgetragene Methode der Sym- 


\section{physeotomie die offene, bei der zuerst der Mons veneris und dann erst der Symphysenknorpel durchschnitten wird.}

Die Geschichte der Schambeintrennung ist kurz folgende: wir folgen dabei A. Döderlein (Über Vergangenheit und Gegenwart der Geburtshülfe [1897]): Wie dieser bemerkt, sind die Urteile wohl über keine andere Operation in verschiedenen Zeiten so schwankend gewesen, wie gerade bei dieser. Schon im Anfang des 17. Jahrhunderts wurde dieser Weg der Beckenerweiterung von Severin Pineau der Pariser Académie de Médecine vorgeschlagen, aber erst 1765 von Jean René Sigault ein bestimmter Operationsplan dieser Versammlung vorgelegt. 1777 führte dieser bei Nacht im Privathause unter erschwerenden Umständen eine Schambeintrennung aus und erzielte ein lebendes Kind, und auch die Mutter blieb am Leben. Diese Nachricht wurde mit Begeisterung aufgenommen: Aber bald machte sich ein Umschwung der Meinung geltend, als der berühmte Jean-Louis Baudelocque (1746-1810) als Führer der Opposition auftrat und durch Leichenversuche zeigte, daß die Erweiterung des Beckens oder besser die Verlängerung des geraden Durchmessers des Beckeneinganges durch diesen Eingriff nur sehr gering ausfallen könne.

Im Gegensatz dazu trat der Holländer Peter Camper (1722-1789) warm für sie ein; dennoch verlor sie immer mehr an Boden und 1845 schrieb Carl Caspar von Siebold: «Zeit und Erfahrung haben über eine Operation den Stab gebrochen, die jetzt nur noch verblendeten und tollkühnen Fachgenossen mehr sein kann, als eine historische Merkwürdigkeit.»

Die Resultate waren aber auch danach: Unter 80 bis zum Jahr 1858 veröffentlichten Fällen starben 28 Mütter, also $35 \%$; von den Kindern kamen 47, also 58,75\% trotz der Operation, die sie retten sollte, ums Leben.

Im Jahre 1881 trat Morisani auf den Plan mit einer warmen Befürwortung der Symphyseotomie. Er verlor unter 50 Operierten nur $20 \%$ Mütter und $18 \%$ Kinder. Die Antiseptik feierte auch hier einen Triumph! Pinard (1844-1934) in Paris, in Deutschland W.A.Freund, Leopold, Zweifel wandten sich dieser Operation zu und veröffentlichten ziemlich günstige Resultate.

Dennoch hatte die Symphyseotomie Mühe, sich allgemein durchzusetzen: man scheute die damit verbundenen Gefahren, vor allem die Blutung und die schwierige Behandlung dieser komplizierten Beckenwunde. Bei schlechter Heilung der Knorpelfuge blieb ein watschelnder Gang als Ausdruck des gesprengten Beckenringes zurück. Auch konnte nur wenig Platz gewonnen werden, wenn man nicht riskieren wollte, durch zu große Distanz der Symphysenenden die Kreuzhüftbeinfugen zu zerreißen.

Unerwartet aber trat eine Konkurrenzoperation auf, die Pubiotomie, oder wie sie dann später genannt wurde die Hebosteotomie. Es wurde vorgeschlagen, statt des Symphysenknorpels den Knochen neben der Symphyse zu durchtrennen. Der erste, der diesen Eingriff, allerdings ungewollt ausführte, weil er die Symphyse nicht zu trennen vermochte, war Carl Caspar von Siebold in Würzburg im Jahre 1778. Aitken empfahl wenige Jahre später seine Methode als grundsätzliche Modifikation: trotzdem er sie mit einer von ihm erfundenen Kettensäge zu machen vorschlug, verliefen mehrere Fälle, die Galbiati ausführte, unglücklich. Der Aitkensche Vorschlag lag darin, daß er beidseitig den horizontalen und den absteigenden Schambeinast zu durchtrennen empfahl, um dann bei der Einheilung der so mobilisierten Symphyse etwas weiter nach vorne, wenn möglich eine definitive Erweiterung des Beckeneinganges zu erreichen. 
Champion 1821 schlug die einseitige Pubiotomie vor; Stoltz demonstrierte 1838 am Kadaver die subcutane Operation. Mittels einer durch eine kleine Öffnung am Schambeinkamm eingeführten gekrümmten Nadel sollte eine Kettensäge hinter der Symphyse durchgezogen und der Knochen von hinten nach vorne durchtrennt werden. Seine Methode wurde nie an den Lebenden geprüft; ebensowenig der Vorschlag Carbonais vom Jahre 1841, die Symphyseotomie subcutan zu machen, indem durch eine kleine Weichteilwunde am oberen Rande ein Bistouri hinter die Symphyse geschoben und diese von hinten nach vorne durchschnitten werden sollte.

Alle die verschiedenen Vorschläge zeigten das Interesse an diesen Eingriffen; aber die praktischen Versuche fielen unglücklich aus. Aitken verfiel zudem dem Wahnsinn, was v. Siebold veranlaßte, seinen Vorschlag als dem Wahnsinn entsprossen zu bezeichnen.

Und doch erlebte die Pubiotomie eine neue Auferstehung als Gigli ihm 1894 empfahl, grundsätzlich die Pelviotomie statt der Symphyseotomie zu machen, wofür er eine Drahtsäge erfunden hatte, die als sehr einfach und handlich, auch sonst in der Chirurgie mannigfache Anwendung gefunden hat. Bonardi machte die erste solche Drahtsägeoperation mit gutem Erfolg, und bald häuften sich die günstigen Berichte. Besonders warm trat van de Velde dafür ein. Alle diese Operationen wurden aber offen, d.h. unter Durchtrennung der Weichteile gemacht. 1903 erst kam Döderlein auf die Idee, die Hebosteotomie, wie sie von van de Velde getauft wurde, subcutan auszuführen. Er suchte dadurch die Verletzung der Blutgefäße in der Gegend vor und hinter der Schamfuge zu vermeiden. Mit einem dazu bestimmten Instrument, einer krummen Nadel mit Griff, ging er von einem kleinen Schnitt am Schamfugenrand aus und unter Schutz eines in diese eingeführten Fingers hinter und hart an der Symphyse nach unten und durchtrennte die Haut nur stichförmig da, wo die Nadel herauskam. Die Drahtsäge wurde in das Ör der Nadel eingehängt, durchgezogen und mit ihr das Schambein seitlich von der Symphyse durchsägt.

Diese Operationen wurden nun öfters ausgeführt und eine Statistik von Schläfli 1909 zeigte bei 664 Fällen eine mütterliche Mortalität von $4,82 \%$ und eine kindliche von $9,81 \%$.

Stets war aber bei diesen Eingriffen, wie schon gesagt, eine Verminderung der Beckenfestigkeit bis zu beweglich bleibender Schamfuge und Gehstörungen ernster Art zu befürchten und, wie $v$. Herff uns einmal am Ärztetag in Basel 1908, wo er eine Operierte vorstellţe, sagte: timeo herniam, er fürchtete, daß durch die Knochenlücke, die sich schlecht schließen ließ, eine Hernie sich durchdrängen würde.

Auch die Versuche, durch Schrägschnitt durch den Knochen und Heilung bei dadurch weiter gestelltem Becken eine definitive Erweiterung des Einganges zu erwirken, haben sich wenig bewährt.

Heute ist die Symphyseotomie und die Hebosteotomie zu einer nur spärlich mehr ausgeführten Operation geworden. Der durch die verbesserten Operationsmethoden und die Aseptik recht lebenssicher gewordene Kaiserschnitt, die Schnittentbindung durch die Bauchdecken, hat ihr den Rang abgelaufen.

R. v. Fellenberg (Bern). 